\title{
POLÍTICAS DE CIÊNCIA, TECNOLOGIA E INOVAÇÃO NO BRASIL: PANORAMA HHISTÓRICO E CONTEMPORÂNEO
}

\author{
Leilah Santiago Bufrem \\ Universidade Federal de Pernambuco - UFPE \\ santiagobufrem@gmail.com \\ Murilo Silveira \\ Universidade Federal de Pernambuco - UFPE \\ muriloas@gmail.com
}

Juliana Lazzarotto Freitas

Universidade Estadual Paulista (UNESP) Campus Marília

julilazzarotto@gmail.com

\begin{abstract}
Resumo
Destaca, a partir da década de 1970, no Brasil, a ênfase ao desenvolvimento científico e tecnológico, em sua demanda por mecanismos e políticas para regular padrões de qualidade e avaliar a ciência produzida. O cenário da informação foi mudando de prioridades devido ao desenvolvimento das tecnologias de informação e de comunicação, inclusive com a consolidação da Ciência da Informação no mundo, priorizando a infraestrutura tecnológica de informação. Essa mudança de paradigma, em 1990, desponta com um conjunto de recursos de infraestrutura tecnológica demandados para o funcionamento de organizações, sociedades e atividades, paralelamente ao advento da chamada sociedade da informação, com seu viés tecnicista e estritamente mercadológico em inovação. Menciona algumas implicações decorrentes dessas mudanças, como a assimetria geográfica no desenvolvimento da pesquisa e inovação. Destaca as agências fomentadoras e reguladoras das políticas públicas de CT\&I que hoje se destacam pelas ações e iniciativas relacionadas à ciência: MCTI, CNPq e CAPES. Esta última estabelece os parâmetros e o processo de avaliação dos periódicos e dos programas de pósgraduação brasileiros.
\end{abstract}

Palavras-chave: Políticas Públicas. Ciência, Tecnologia e Inovação. Política em Ciência. Tecnologia e Inovação. Brasil. História.

\section{SCIENCE, TECHNOLOGY, AND INNOVATION POLICIES IN BRAZIL: HISTORICAL AND CONTEMPORARY FRAME}

\begin{abstract}
It presents, from the 1970s, in Brazil, the emphasis on scientific and technological development, which its demand for mechanisms and policies to regulate quality standards and evaluate the science produced. The information frame was changing priorities due to the development of information and communication technologies, including the consolidation of the Information Science in the world - giving priority to information technology infrastructure. This paradigm shift, in 1990, emerges with a set of technological infrastructure resources demanded for the functioning of organizations, societies and activities, in parallel with the advent of the so-called information society, with its technicist bias and strictly market in innovation. Some of the implications of these changes have been mentioned, such as the geographical asymmetry in the development of research and innovation. The agencies promoting and regulating public policies in CT\&I that stand out, today, for actions and initiatives related to science are: MCTI, CNPq and CAPES. This last establishes the parameters and the process of evaluation of the periodicals and the Brazilian postgraduate programs.
\end{abstract}

Keywords: Public Policy. Science, Technology and Innovation. Policy in Science. Technology and Innovation. Brazil. History.

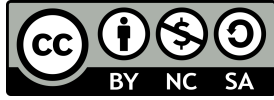

P2P \& INOVAÇÃO, Rio de Janeiro, v. 5 n. 1, p.6-25, Set./Fev. 2018. 


\section{INTRODUÇÃO}

Também chamada de Política em Ciência, Tecnologia e Inovação (CT\&I), a política científica e tecnológica constitui objeto de estudo complexo e que permite ampla variedade de recortes. Essa complexidade, confirmada pela controversa produção bibliográfica sobre o tema, os conceitos e as formas de avaliação de políticas públicas, exige que se tome como categorias para reflexões iniciais, seus elementos constitutivos: a) mecanismos de transmissão que envolvem as atividades de CT\&I e sua articulação com o desenvolvimento econômico e social; b) fixação de um conjunto de objetivos e diretrizes com base nessa interpretação; c) adoção de um conjunto de instrumentos visando alcançar os objetivos estabelecidos (CAVALCANTE, 2009).

As decisões no campo científico sempre envolvem a dimensão política, pois as escolhas feitas pelos agentes fundamentam-se em interesses pessoais ou institucionais, ou, ainda, em atos intencionalmente voltados aos modos de produção ou reprodução na ordem social estabelecida. Alguns exemplos de temáticas que compõem o escopo relacionado a esse objeto são programas de pesquisa, instrumentos de financiamento, instituições, legislações e a dinâmica de geração de conhecimento e de inovação no campo científico (DIAS, 2011).

A concepção de política científica e tecnológica enunciada por Dias (2011) a situa como produto da tensão existente entre a "agenda da ciência", o conjunto de interesses relativamente articulados da comunidade de pesquisa e "as agendas da sociedade", que envolvem uma pluralidade de atores e interesses.

Surgem, a partir dessas forças, expectativas diferenciadas em relação ao apoio ao fazer científico contemporâneo. Entretanto, a atual conjuntura científica no Brasil revela o amadurecimento da postura crítica em relação ao que se está produzindo, especialmente, quando se percebe a ampliação de estudos metacientíficos ou de avaliação dos modos de se fazer e compreender a ciência.

Face ao panorama descrito, alguns questionamentos impõem-se como necessários para entender diacronicamente as funções dessas políticas na atual conjuntura científica e tecnológica no Brasil: a) como se configura o contexto histórico e contemporâneo das agências brasileiras reguladoras e de fomento à pesquisa? b) qual o papel dessas agências e de que modo contribuem para o desenvolvimento da pesquisa no Brasil, junto às instituições produtoras do conhecimento científico?

A partir desses questionamentos, define-se como objetivo geral deste estudo apresentar um panorama das Políticas de Ciência, Tecnologia e Inovação no Brasil, contextualizando o 
sistema de comunicação científica em suas relações com as iniciativas nacionais das agências reguladoras responsáveis pelo planejamento e aplicação destas políticas. Especificamente, procura-se: a) analisar o contexto histórico e contemporâneo das agências brasileiras reguladoras e de fomento à pesquisa; b) apontar o papel dessas agências e seus modos de contribuição para o desenvolvimento da pesquisa no Brasil; c) perceber como atuam as agências avaliadoras e seus sistemas de avaliação junto às instituições produtoras do conhecimento científico.

\section{CONTEXTO HISTÓRICO E CONTEMPORÂNEO DAS POLÍTICAS E AGÊNCIAS BRASILEIRAS}

Concebendo a política de informação científico-tecnológica como um conjunto de princípios e escolhas que direcionam os modos de geração, uso e absorção de ICT, González de Gómez e Canongia (2001) argumentam que ela se realiza por meio de diferentes procedimentos de promoção, regulação, coordenação e articulação. E mais, ela interage com as "condições resultantes das políticas, práticas e contextos daprodução de ciência, tecnologia e inovação e das formas socialmente estabelecidas de sua apropriação" (GONZÁLEZ DE GÓMEZ, 2001, p. 15). De outro ponto de vista, pode ser esclarecida como um conjunto de leis, regras, práticas e orientações sob as quais a pesquisa científica é conduzida. Ademais, envolve aspectos que influenciam seu arcabouço legal-regulatório e como ele é desenhado e implementado (DIAS, 2011).

Uma especificidade nem sempre reconhecida da política científica e tecnológica é seu caráter de política-meio. Como tal, essa categoria de política, ao menos teoricamente, configura-se como suporte para as demais políticas públicas em outros setores, como por exemplo, o industrial, o agrícola, o educacional, o da saúde, o da inclusão social, entre outros. Portanto, embora tenha objetivos próprios, esses constituem elementos de uma estratégia maior, geralmente, atrelada a outras políticas (DIAS, 2011).

Além disso, considera-se que uma política de CT\&I deve ter sua ênfase na excelência científica e tecnológica, pautada na consolidação de uma indústria inovadora e na capacidade de enfrentar os desafios que lhe são impostos com conhecimento em todos os setores.

A possibilidade de análise do comportamento das comunidades científicas tem sido defendida por Cole e Cole (1968), ao argumentarem que o bom funcionamento do sistema de comunicação seria a condição operacional do sistema de recompensas, o que se pode concluir como decorrência natural do processo de avaliação. Por ele se distinguem a visibilidade 
(visibility), ou capacidade de ser visto, da capacidade de reconhecer o outro (awareness) e aperfeiçoam-se os critérios de qualidade em relação à produção científica, aos departamentos e às instituições, aos quais estão vinculados as produções e os autores.

As políticas que vêm sendo adotadas nas instituições de ensino superior de qualquer natureza têm sido fortalecidas no Brasil por agências de pesquisa, as quais, ao institucionalizarem normas e regulamentos, estimulam sistemas de avaliação e parâmetros para evidenciar as meritocracias científicas. Assim dimensionado, o investimento em ciência, tecnologia e inovação tem contado com o apoio de pensadores da sociedade organizada, que o percebem como o meio por excelência para impulsionar o crescimento econômico, gerando empregos e desenvolvendo as habilidades daqueles que fazem parte do processo de produção e comunicação do conhecimento científico.

Tanto lideranças acadêmicas e políticas, quanto gestores de organizações, instituições ou grandes corporações, em diversos países, vêm convergindo em direção ao entendimento de que a pesquisa é um investimento fundamental para o desenvolvimento sustentável e para a melhoria da qualidade de vida dos povos.

Para cumprir a proposta de apresentar breve panorama das políticas de CT\&I no Brasil, procura-se contextualizar de que forma o sistema de comunicação científica mantém-se a partir de políticas e iniciativas nacionais, identificando as agências de fomento e reguladoras, para reconhecer as ações que têm contribuído para o desenvolvimento nacional e que têm valorizado o avanço do conhecimento e da inovação.

Com esse propósito, inicialmente, apresenta-se breve histórico sobre quando e como surgiu a preocupação com o desenvolvimento das políticas de CT\&I no país; como decorreu a criação das principais agências brasileiras reguladoras e de fomento à pesquisa, assim como seu papel na sociedade, procurando-se esclarecer a influência das políticas estabelecidas pelas agências para as universidades e demais instituições de pesquisa.

Adiante, procura-se reconhecer e analisar o papel das agências de fomento e de que modo elas contribuem para o desenvolvimento da pesquisa no Brasil, com exemplos de iniciativas relacionadas à CT\&I. Em seguida, apresenta-se a atuação da agência avaliadora nacional do ensino superior, a CAPES, e seu sistema de avaliação junto às instituições produtoras do conhecimento científico.

Respondendo-se à questão "Como, quando e por que surgiram as primeiras preocupações relacionadas às políticas CT\&I no Brasil?” pode-se dizer que elas emergem no cenário pós-guerra, devido ao aumento da produção científico-tecnológica, que aponta a necessidade da informação como insumo para transformar conhecimento em bens e serviços. 
Como fator estratégico, as políticas de CT\&I já surgem vinculadas às políticas de informação e vêm sendo delineadas historicamente mediante a intervenção do Estado.

Em relação às etapas do processo histórico de organização das atividades de ciência no país, mais especificamente, da criação das bases para uma Política Nacional de Ciência e Tecnologia, um dos atores do processo foi a Organização das Nações Unidas para a Educação, Ciência e Cultura (ONU), que estimulou o estabelecimento de instituições, sistemas e políticas nacionais de informação científica e tecnológica em diversos países (SILVA; GARCIA, 2014).

A histórica relação entre informação e política foi estabelecida por sua inclusão na esfera de intervenção do Estado, tanto como dimensão de racionalidade administrativa, quanto como fator estratégico do desenvolvimento científico-tecnológico (GONZÁLEZ DE GÓMEZ, 2002).

As décadas de 50 a 70, do século XX, foram marcadas pela criação dos organismos que se incumbiram da formulação das políticas públicas no Brasil e pelo início da reorganização das atividades de ciência e tecnologia no país. Nesse período, foram criados órgãos decisivos para a consolidação de uma estrutura de CT\&I:

a) Conselho Nacional de Pesquisa (CNPq)- 1951;

b) Coordenação de Aperfeiçoamento de Pessoal de Nível Superior (CAPES) -1951;

c) Instituto Brasileiro de Bibliografia e Documentação (IBBD) - 1954;

d) Sistema Nacional de Informação Científica e Tecnológica (SNICT)- 1971;

e) Instituto Brasileiro de Informação em Ciência e Tecnologia (IBICT) - 1976.

Podem ser considerados outros marcos históricos que influenciaram esse processo de formulação de políticas científicas no Brasil, a saber:

a) criação de sistemas de informação na área de saúde como o Centro Latino-Americano e do Caribe de Informação em Ciências da Saúde (BIREME) e na área agrícola, como a Empresa Brasileira de Pesquisa Agropecuária (EMBRAPA);

b) organização do Programa de Comutação Bibliográfica (COMUT);

c) expansão do ensino superior;

d) criação dos primeiros cursos de pós-graduação stricto sensu;

e) criação do Institute for Scientific Information (ISI) e do Science Citation Index (SCI) por Garfield, influenciados pelas atividades realizadas pelo Instituto de Informação Técnica e Científica (VINITI), Rússia.

Cria-se, desse modo, ambiente propício para pesquisas com demanda para a formação de profissionais mais críticos na área de informação.

Deve-se esclarecer que, em 1976, o IBBD se tornou Instituto Brasileiro de Informação em Ciência e Tecnologia (IBICT), órgão nacional de informação, como unidade de pesquisa do 
Ministério da Ciência, Tecnologia e Inovação (MCTI), priorizando o campo da Ciência da Informação e temas relacionados. Sediado em Brasília, conta com uma coordenação de pesquisa e pós-graduação na sede do Centro Brasileiro de Pesquisas Físicas, junto ao campus da Praia Vermelha, da Universidade Federal do Rio de Janeiro (UFRJ), no bairro da Urca, Rio de Janeiro.

O Instituto vem acompanhando e internalizando as tecnologias de informação e de comunicação e estimulando o empreendedorismo e a inovação. Com o apoio dessas tecnologias, vem assumindo o papel de agregador e integrador das iniciativas de informação científica e tecnológica no país. Desde 1976, assumiu as funções do SNICT, extinto no período, o que reforçou ainda mais sua atuação como executor de políticas de desenvolvimento científico e tecnológico. Destaca-se que o IBICT tem contribuído expressivamente para a institucionalização da área de Ciência da Informação no país, sendo que uma de suas contribuições foi a criação do primeiro programa de pós-graduação da área.

A implantação de políticas na atual conjuntura brasileira resultou na descontinuidade desses planos e políticas, refletindo-se em algumas de suas deficiências, acompanhando a história das políticas de CT\&I no país, conforme o desenvolvimento das forças produtivas desde o Brasil Colônia e, nesse processo, a organização espacial da ciência e tecnologia segue o mesmo padrão de localização do setor produtivo, com uma estrutura polarizada (ARAÚJO, 1985).

Em detrimento da implementação da infraestrutura tecnológica nacional para desenvolvimento dos planos e política de informação, como ocorreu nos países desenvolvidos como Estados Unidos e Canadá, para a consolidação dos campos de informática, redes e telecomunicações, partindo então da Pesquisa e Desenvolvimento (P\&D) para a indústria e promoção de serviços (SILVA, 2005), no caso do Brasil houve um movimento no sentido inverso, abrindo os serviços de telecomunicações para "encurtar o período de acomodação à nova realidade", ou seja, priorizou-se o desenvolvimento da infraestrutura para a prestação de serviços e a indústria, chegando-se à P\&D como resultado. Portanto, "grande parte da infraestrutura tecnológica utilizada na implantação da sociedade da informação" [...] em países como Brasil advêm dos núcleos norte-americanos de pesquisa (SILVA, 2005, p. 24).

Nesse sentido, a conjuntura definida para a implementação das políticas de informação científica e tecnológica tem refletido no estabelecimento do que se denominou sociedade da informação, especialmente a partir da década de 1990. A motivação de iniciativas nacionais nessa linha, segundo Takahashi (2000, p. 108), teve "forte viés econômico." 
Analisar a trajetória das políticas em ciência e tecnologia no Brasil ao longo das últimas décadas é desafiante, pois além de ser tarefa complexa devido aos múltiplos fatores que a influenciam, é uma fonte de reflexão sobre a dinâmica de elaboração de políticas públicas num contexto periférico. Esse aspecto, conforme Dias (2012), é ignorado pela literatura produzida nos países centrais.Pode-se afirmar que os problemas que se verificam, hoje, no processo de formulação, planejamento, aplicação e avaliação das políticas de CT\&I no país, bem como a "infraestrutura tecnológica para informação e comunicação, cuja dissonância restringe a interoperabilidade de sistemas de informação" (FREITAS, BUFREM, 2016, não paginado), são produto de um processo histórico de consolidação das políticas de informação no país.

$\mathrm{Na}$ maioria dos países em desenvolvimento, em especial os da América Latina, ainda não foi possível estruturar "coalizões políticas capazes de equacionar minimamente a questão de como desenhar políticas públicas capazes de impulsionar o desenvolvimento econômico" (SOUZA, 2006, p. 21) e que, ao mesmo tempo, visem a inclusão social.

O histórico de desenvolvimento das políticas de CT\&I no país contempla aspectos que contribuíram para a estruturação do campo da Ciência da Informação. A institucionalização da CI foi marcada pela aproximação de pesquisadores e profissionais de outras áreas que necessitavam resolver seus problemas de informação e comunicação.

Nesse sentido, segundo Freitas e Bufrem (2016), as questões relacionadas à produção, organização e comunicação da ciência foram mais evidentes nas ciências que tinham maior representatividade e prestígio no processo de desenvolvimento científico e tecnológico do país, com ênfase para a área agrícola e de saúde, cuja produção científica e técnica se ampliava significativamente, em especial, com a criação de sistemas de informação nestas áreas, como a EMBRAPA e a BIREME (LIMA, 1993; PIRES-ALVES, 2008).

Logo, as áreas mais desenvolvidas na produção e na comunicação da ciência acabam por exercer mais esforços em prol das políticas de CT\&I, especialmente porque recebiam e seguem recebendo mais fomento para isso.

Ao considerar que a informação é agente de transformação social, enfatiza-se o papel dos profissionais e cientistas da informação para a discussão de questões relacionadas às políticas de CT\&I.

Considera-se que a práxis da área vai além dos aspectos da produção, organização e comunicação da ciência, voltando-se também às políticas sociais, contribuindo concretamente com a produção, gestão e avaliação das políticas, promovendo o acesso à informação, à inclusão social, e com o desenvolvimento de uma sociedade mais crítica. Os pesquisadores e profissionais da Ciência da Informação podem atuar no âmbito das políticas públicas de ciência 
e tecnologia de distintas maneiras e em diversas etapas do ciclo de políticas, conforme se apresenta na Figura 1.

Figura 1: A Ciência da Informação atuante no ciclo de políticas públicas de CT\&I.

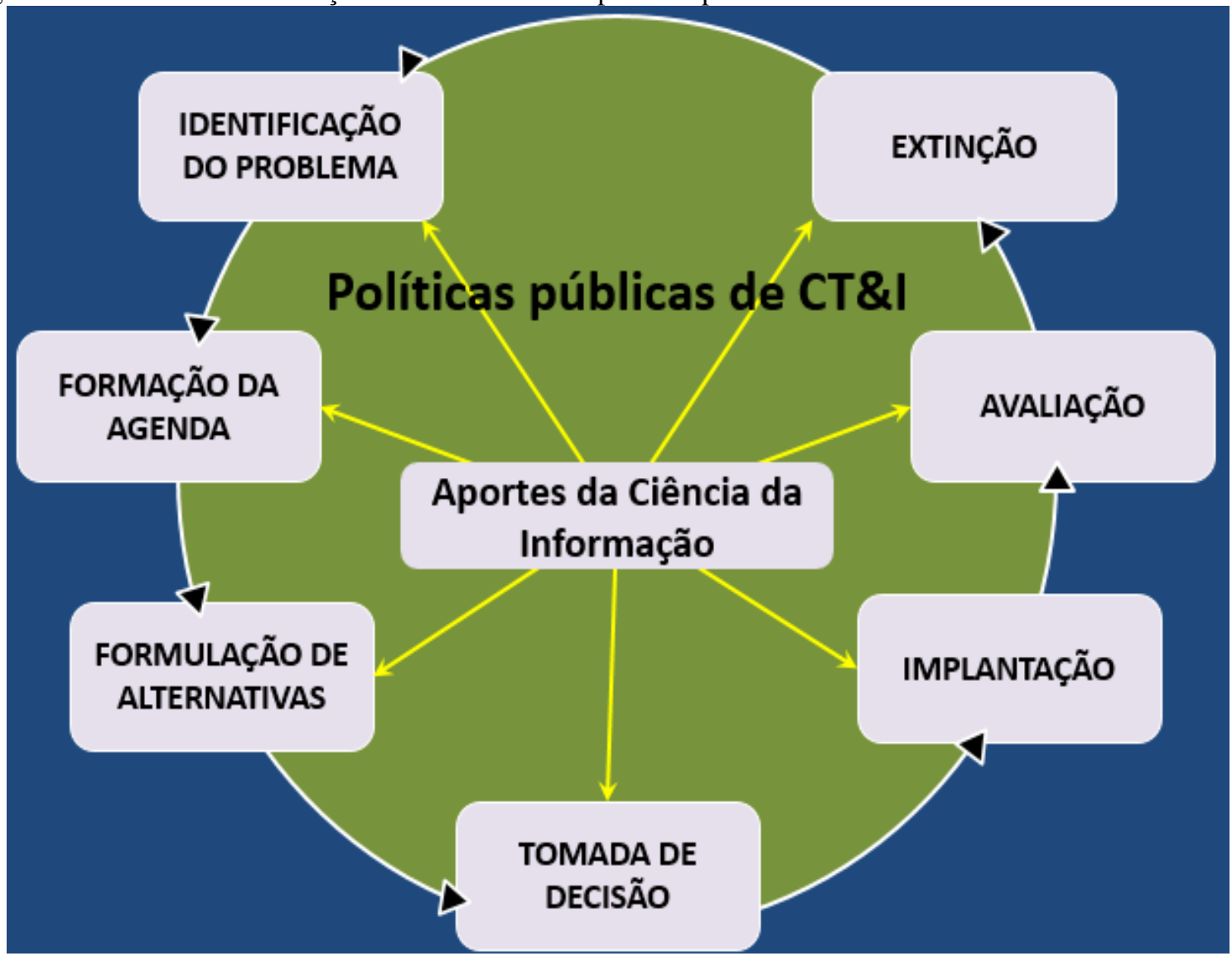

Fonte: Adaptação de Howlett e Ramesh e Perl, 2013.

As políticas públicas configuram-se em processos não somente complexos, mas sinalizadores de construtos políticos característicos da contemporaneidade (JARDIM; SILVA; NHARRELUGA, 2009). Portanto, quando se trata de políticas científicas diretamente voltadas ao aperfeiçoamento da gestão e avaliação da produção científica, esse espaço para o cientista da informação parece se ampliar, especialmente porque a CI contempla vastos conhecimentos relacionados aos estudos métricos da informação e às análises de domínio, essenciais para o aprofundamento e compreensão relacionais de dados bibliométricos sobre campos científicos específicos. Destaca-se a crítica realizada por Frohmann (1995) como uma das limitações daliteratura sobre política pública, que seria a ausência de ênfase na relação entre informação e poder, ou seja, sobre como o poder é exercido através de relações sociais mediadas pela informação.

Ao estudar políticas públicas, alerta-se para a compreensão de que elas representam um conjunto de decisões e medidas efetivas, decorrentes das prioridades estabelecidas a partir de 
uma realidade concreta. Essas medidas, embora sejam consideradas por sua natureza pública, não são perceptíveis apenas nas ações do Estado, cuja dimensão é facilmente observável, mas também decorrem doque o Estado deixa de decidir ou realizar e das forças latentes da sociedade. Além disso, as características do contexto social são fatores determinantes para processos e decisões políticas internas ao Estado, o que afeta necessariamente as relações de poder exercidas, as contradições e os conflitos entre classes sociais, típicos das sociedades capitalistas.

A partir da necessidade de criação de condições teóricas e contextuais que promovam as realizações concretas, inclui comportamentos, intenções, decisões, de natureza mais ou menos autoritária, que estão inscritas em um quadro geral de ação e apresentam público e objetivos definidos" (JARDIM; SILVA; NHARRELUGA, 2009, não paginado). Logo, somente há política pública quando as ações estudadas formam um todo coerente com o regime econômico e político vigente no país. Quem as estuda tem a oportunidade de perceber o grau de persistência e consistência da democracia que as envolve. A política só pode ser gerada por interações entre decisões "mais ou menos conscientes de diversos atores sociais, e não somente dos tomadores de decisões.

Muitas vezes, o que é denominado pelo próprio Governo de política pública não se configura como tal, mas sim como ações, programas e projetos governamentais sem compromisso em monitorar e avaliar seu impacto (JARDIM; SILVA; NHARRELUGA, 2009). Também se observam dificuldades no estabelecimento de indicadores de ciência e tecnologia, pois a área abarca um espectro de atividades com fins e demandas muito diferentes. Ademais, a perspectiva de longo prazo das ações em CT\&I dificulta a avaliação e interpretação de seus resultados, especialmente no que tange aos recursos intangíveis para mensuração (BRASIL, 2012b).

Os indicadores de CT\&I objetivam “[...] fornecer informações especializadas à comunidade científica, aos formuladores de políticas aos legisladores, à imprensa, aos alunos e à sociedade em geral" (BRASIL, 2012a). Também, agregam dados de diversas fontes para prover uma visão global do sistema nacional de Ciência, Tecnologia e Inovação e seus diversos atores, ligados ou não ao Governo Federal, em suas várias dimensões, permitindo a comparação com outros países e a realização de análises variadas das políticas de CT\&I.

Após esse histórico relativo ao Sistema Nacional de Informação em Ciência e Tecnologia, apresenta-se, na sequência, a descrição das principais agências brasileiras reguladoras e de fomento à pesquisa e suas funções, a fim de tratar de que modo influenciam as políticas estabelecidas pelas agências para as universidades e demais instituições de pesquisa. 


\section{AGÊNCIAS DE FOMENTO}

O planejamento e a implementação das políticas setoriais no país têm adotado como referência documentos voltados aos desafios diagnosticados e às estratégias mais relevantes para cumprir a política nacional de cada setor. Para tanto, são elaborados planos e documentos setoriais específicos.

Grupos especializados, em permanente interlocução com os principais atores setoriais e regionais, produzem documentos específicos sobre as políticas de cada um dos setores, em consonância com as diretrizes mais amplas de Governo e com as especificidades legais e políticas relacionadas. Assim, são construídos os planos setoriais e regionais, cujas iniciativas são planejadas e definidas também em acordo com a conjuntura mobilizadora dos atores, dos recursos, em prol dos resultados pretendidos.

Embora se incorporem novos atores ao processo decisório das Políticas em Ciência e Tecnologia, sua agenda ainda segue sob o controle da comunidade de pesquisa, responsável pela formulação, implementação e avaliação dessa política (DIAS, 2011).

Dela depende a definição de uma estratégia nacional de desenvolvimento científico e tecnológico, a ser fundamentada em princípios de justiça e de equidade social, com o uso intencional da ciência, além do desenvolvimento e da disseminação de tecnologias apropriadas à conjuntura local. Destacam-se, nesse processo, temas centrais para o desenvolvimento da CT\&I do país, considerando-se tanto os avanços recentes nas políticas do setor, como os desafios mais relevantes para o fortalecimento do Sistema Nacional de CT\&I.

Alguns objetivos das políticas científicas na contemporaneidade tangem, especialmente, à avaliação da pós-graduação nacional; aos subsídios do Ministério da Educação na definição dos planos e políticas de desenvolvimento da pós-graduação nacional; à formação de recursos mediante programas de investimento em bolsas de estudo; aos auxílios e outros mecanismos, como promoção da cooperação científica nacional e internacional e viabilização do acesso à produção científica. É nesse cenário que se menciona o papel de algumas agências de fomento para o desenvolvimento da pesquisa no Brasil.

Por meio deste aprofundamento em políticas públicas, nota-se que o impacto ideal de uma agenda de desenvolvimento científico e tecnológico deve ser intelectual - diretamente relacionado às transformações na ciência; social - afetando a população; e econômico movimentando e aperfeiçoando os serviços e produtos nos distintos setores industriais. Entretanto, a ênfase que se observa atualmente tem sido maior na obtenção de financiamentos e na inovação, com a supervalorização de resultados quantitativos na atividade de pesquisa, 
sem o olhar para todas as finalidades e dimensões envolvidas no desenvolvimento da ciência, tecnologia e inovação no país.

Certas políticas de ciência e tecnologia definidas por agências fomentadoras serão aqui mencionadas para melhor entendimento do processo de avaliação e fomento da atividade científica.

Considerando as principais agências brasileiras de fomento e avaliação da pesquisa, a CAPES e o CNPq já mencionados; a Financiadora de Estudos e Projetos (FINEP); e as Fundações de Amparo à Pesquisa (FAP's), aqui se procura descrever suas funções para que seja reconhecida a influência desse sistema no país.

A dimensão política desdobra-se em ações desenvolvidas pela CAPES, com destaque para o mencionado Portal de Periódicos, criado em 2000, como resposta à precariedade de acesso das bibliotecas brasileiras à informação científica internacional, cumprindo o objetivo de reduzir as desigualdades regionais no acesso à produção científica no país, promovendo o acesso irrestrito de seu conteúdo e a capacitação dos usuários (professores, pesquisadores, alunos e funcionários) para o uso do acervo em suas atividades de ensino, pesquisa e extensão (CAPES, 2017a).

A diversificação de conteúdo do Portal, com aquisição de diferentes títulos e bases, buscou atender à emenda de pesquisa e aos interesses da comunidade científica brasileira. Também foi ampliado o número de instituições usuárias do Portal de Periódicos, conforme critérios de excelência acadêmica e científica definidos pelas CAPES e pelo Ministério da Educação.

O prêmio Capes Tese, criado em 2005, é um produto de iniciativas de valorização da CT\&I, que tem sido concedido anualmente às melhores teses de doutorado defendidas e aprovadas nos cursos reconhecidos pelo MEC, considerando critérios de qualidade e originalidade (CAPES, 2017b).

Outra iniciativa resultante de um esforço conjunto do Ministério da Ciência, Tecnologia e Inovação (MCTI) e do Ministério da Educação (MEC), por meio de suas respectivas instituições de fomento (CNPq e CAPES), Secretarias de Ensino Superior e de Ensino Tecnológico do MEC foi a criação do "Programa Ciência Sem Fronteiras", em 2011, para promover a consolidação, expansão e internacionalização da ciência e tecnologia, da inovação e da competitividade brasileira por meio do intercâmbio e da mobilidade Internacional. O programa objetivou a promoção da inserção internacional das instituições brasileiras pela abertura de oportunidades semelhantes para cientistas e estudantes estrangeiros; o investimento na formação de pessoal altamente qualificado nas competências e habilidades necessárias para 
o avanço da sociedade do conhecimento, o aumento a presença de pesquisadores e estudantes de vários níveis em instituições de excelência no exterior e a ampliação do conhecimento inovador de pessoal das indústrias tecnológicas, atraindo jovens talentos científicos e investigadores altamente qualificados para trabalhar no Brasil (PROGRAMA CIÊNCIA SEM FRONTEIRAS, 2017).

Considera-se relevante mencionar, também, o Programa Nacional de Plataformas do Conhecimento (PNPC), criado em 2014, com a intenção de estimular a pesquisa na área de ciência, tecnologia e inovação (BRASIL, 2017b).

Já o Instituto Brasileiro de Geografia e Estatística (IBGE), com o apoio da FINEP e do Ministério da Ciência, Tecnologia e Inovação desenvolveu, no ano 2000, a Pesquisa de Inovação (PINTEC), com o objetivo de ampliar o potencial analítico dos resultados da Pesquisa de Inovação Tecnológica, a partir do cruzamento de seus dados com os dados de outras fontes estatísticas. Criou-se uma base para gerar indicadores setoriais nacionais e, no caso da indústria, também regionais, das atividades de inovação das empresas brasileiras, comparáveis com as informações de outros países.

O propósito de aumentar a confiabilidade e reprodutibilidade dos resultados de pesquisas publicados tem gerado uma crescente demanda por transparência e abertura na pesquisa e sua comunicação. Em 2009, o Ministério da Ciência, Tecnologia e Inovação (MCTI) lançou o "Mapa de Ações Sociais", ferramenta de consulta pública que visa maior limpidez na utilização de seus investimentos e recursos no âmbito CT\&I para o desenvolvimento social. Essa clara intenção política em prol da transparência teria como marco histórico e institucional a Lei de Acesso à Informação Pública (Lei n. 12.527, de 18 de novembro de 2011), com o objetivo de assegurar o acesso dos cidadãos às informações dos órgãos públicos. Essa Lei viria a ter um significado especial para o exercício da cidadania, a partir da divulgação de informações de interesse público, independentemente de solicitações. Os projetos políticos científicos e culturais tornaram-se efetivamente públicos e os órgãos de fomento passaram a ter seus editais amplamente divulgados.

Pode-se considerar que o mapa de ações sociais do Ministério seja, efetivamente "um instrumento de transparência das ações desta Pasta no conjunto de políticas voltadas para a inclusão social, sobretudo, no que se refere à difusão da ciência e o acesso ao conhecimento e à tecnologia" (BRASIL, 2012a, não paginado). Além de prestar contas, os conteúdos presentes no instrumento consolidam os compromissos do Plano de Ação: Ciência, Tecnologia e Inovação para o Desenvolvimento Nacional (PACTI 2007-2010). 
Entre as principais ações do CNPq ao longo de sua história, está o fomento à pesquisa, cujos carros-chefes atuais são: a) a Chamada Pública Universal, destinada ao atendimento de projetos de pesquisadores individuais; b) o Pronex, Programa voltado para grupos de excelência, e c) os Institutos Nacionais de Ciência e Tecnologia (INCT's).

O Edital Universal é uma das ações mais efetivas do CNPq, pois representa a demanda espontânea pela pesquisa das universidades e dos centros de pesquisa, uma vez que é aberto aos pesquisadores doutores para a realização de projetos e estudos em todas as áreas do conhecimento. Criado com o objetivo de financiar a execução de projetos apresentados por pesquisadores com contribuição significativa ao desenvolvimento científico e tecnológico do país, a Chamada Universal tem sido exemplo significativo de ação geral que foi bastante ampliada: no período de seis anos, de 2001 a 2006, foram destinados cerca de R \$ 280 milhões, enquanto que nos três anos, de 2007 a 2009, foram R\$ 350 milhões.

Em 2010, o número de projetos aprovados teve um aumento significativo, $31 \%$ maior do que o de 2009, ampliação devida a uma política de acompanhamento da expansão do Sistema Nacional de Ciência e Tecnologia (SNCT). Essa realidade expressa-se no aumento do número de bolsas de iniciação científica (IC), mestrado, doutorado e produtividade em pesquisa (PQ), além do desenvolvimento tecnológico e extensão inovadora (DT), entre outros (CNPq, 2017).

Uma modalidade de fomento de extrema importância para o desenvolvimento da pesquisa é o programa de concessão de Bolsa de Produtividade em Pesquisa, direcionado a pesquisadores de todas as áreas do conhecimento com o objetivo de distinguir seu trabalho e valorizar sua produção. Entre os critérios para a concessão desta modalidade de bolsa, estão a produção científica, a participação na formação de recursos humanos e a efetiva contribuição para a área de pesquisa (CPNQ, 2017). Vale dizer que o SNCT, de 2002 a 2010, incorporou, cerca de 10 mil novos pesquisadores doutores, resultado da expansão das universidades federais e estaduais, institutos federais e institutos de pesquisa.

Além disso, tem-se a Rede Nacional de Ensino e Pesquisa (RPN), que foi criada em 1989 para infraestrutura nacional de rede internet de âmbito acadêmico e que vem evoluindo periodicamente. Hoje, seu grande desafio é promover infraestrutura de internet avançada para o interior, permitindo que alunos, professores, pesquisadores e colaboradores de instituições das áreas de educação, saúde e cultura possam se comunicar e estabelecer colaborações com pares espalhados no país (REDE NACIONAL DE ENSINO E PESQUISA, 2017).

A FINEP, criada em 24 de julho de 1967, é uma empresa pública brasileira de fomento à ciência, tecnologia e inovação em empresas, universidades, institutos tecnológicos e outras instituições públicas ou privadas, sediada no Rio de Janeiro. A FINEP substituiu e ampliou o 
papel até então exercido pelo Banco Nacional de Desenvolvimento Econômico e Social e seu Fundo de Desenvolvimento Técnico-Científico com a finalidade de financiar a implantação de programas de pós-graduação nas universidades brasileiras. A empresa é vinculada ao Ministério da Ciência, tecnologia e inovação.

O Prêmio FINEP, criado em 1998, tem contribuído para o reconhecimento e divulgação de esforços inovadores realizados por empresas, instituições sem fins lucrativos e pessoas físicas, no Brasil, já inseridos no mercado interno ou externo. As empresas, instituições e os inventores são aqueles que desenvolvem soluções em forma de produtos, processos, metodologias e/ou serviços novos ou significantemente modificados. Já premiou centenas de empresas, instituições e pessoas físicas, sendo responsável pela projeção dos contemplados não apenas no Brasil, como no exterior.

As Fundações de Amparo à Pesquisa fazem parte de uma categoria específica de fundação (ou entidade) que viabiliza recursos para o desenvolvimento da pesquisa nas diversas áreas da ciência e tecnologia. Elas se constituem em entidades que integram o Sistema Nacional de Ciência e Tecnologia (SNCT), juntamente com as instituições tradicionais como CNPq e FINEP e exercem papel significativo tanto para as definições, quanto para a execução da política científico-tecnológica nacional.

A primeira das FAP's do Brasil foi fundada, em 1962, no Estado de São Paulo, e se denomina Fundação de Amparo à Pesquisa do Estado de São Paulo (Fapesp). A partir desta, outros Estados brasileiros passaram a estruturar suas próprias fundações, baseados no modelo da Fapesp. Em 1964, o Estado do Rio Grande do Sul fundou a Fundação de Amparo à Pesquisa do Estado do Rio Grande do Sul (Fapergs); em 1980, o Estado do Rio de Janeiro fundou a Fundação de Amparo à Pesquisa do Estado do Rio de Janeiro (Faperj); em 1985, foi fundada a Fundação de Amparo à Pesquisa do Estado de Minas Gerais (Fapemig). Mas o crescimento expressivo das FAP'sem outros Estados deu-se na década de 90. Atualmente, as FAP'spodem ser visualizadas no Quadro 1.

Quadro 1: Fundações Estaduais de Amparo à Pesquisa.

\begin{tabular}{c|c}
\hline ESTADOS & FUNDAÇÕES ESTADUAIS DE AMPARO À PESQUISA \\
Acre & Fundação de Amparo à Pesquisa do Acre \\
\hline Alagoas & Fundação de Amparo à Pesquisa do Estado de Alagoas \\
\hline Amapá & Fundação de Amparo à Pesquisa do Estado do Amapá \\
\hline Amazonas & Fundação de Amparo à Pesquisa do Estado do Amazonas \\
\hline Bahia & Fundação de Amparo à Pesquisa do Estado da Bahia \\
\hline Ceará & Fundação de Amparo à Pesquisa do Estado do Ceará \\
\hline Distrito Federal & Fundação de Amparo à Pesquisa do Distrito Federal \\
\hline Espírito Santo & Fundação de Amparo à Pesquisa e Inovação do Espírito Santo \\
\hline
\end{tabular}




\begin{tabular}{|c|c|}
\hline Goiás & Fundação de Amparo à Pesquisa do Estado de Goiás \\
\hline Maranhão & $\begin{array}{l}\text { Fundação de Amparo à Pesquisa e ao Desenvolvimento Científico e Tecnológico do } \\
\text { Maranhão }\end{array}$ \\
\hline Mato Grosso & Fundação de Amparo à Pesquisa do Estado de Mato Grosso \\
\hline Mato Grosso do Sul & $\begin{array}{l}\text { Fundação de Apoio ao Desenvolvimento do Ensino, Ciência e Tecnologia do Estado } \\
\text { de Mato Grosso do Sul }\end{array}$ \\
\hline Minas Gerais & $\begin{array}{l}\text { Fundação de Amparo à Pesquisa e ao Desenvolvimento Científico e Tecnológico de } \\
\text { Minas Gerais }\end{array}$ \\
\hline Pará & Fundação Amazônia de Amparo a Estudos e Pesquisas do Pará \\
\hline Paraíba & Fundação de Apoio à Pesquisa do Estado da Paraíba \\
\hline Paraná & $\begin{array}{c}\text { Fundação Araucária - Apoio ao Desenvolvimento Científico e Tecnológico do } \\
\text { Paraná }\end{array}$ \\
\hline Pernambuco & Fundação de Amparo à Ciência e Tecnologia do Estado de Pernambuco \\
\hline Piauí & Fundação de Amparo à Ciência e Tecnologia do Estado do Piauí \\
\hline Rio de Janeiro & Fundação Carlos Chagas Filho de Amparo à Pesquisa do Estado do Rio de Janeiro \\
\hline Rio Grande do Norte & Fundação de Apoio à Pesquisa do Estado do Rio Grande do Norte \\
\hline Rio Grande do Sul & Fundação de Amparo à Pesquisa do Estado do Rio Grande do Sul \\
\hline Rondônia & $\begin{array}{c}\text { Fundação Rondônia de Amparo ao Desenvolvimento das Ações Científicas e } \\
\text { Tecnológicas e à Pesquisa do Estado de Rondônia }\end{array}$ \\
\hline São Paulo & Fundação de Amparo à Pesquisa do Estado de São Paulo \\
\hline Santa Catarina & Fundação de Amparo à Pesquisa e Inovação do Estado de Santa Catarina \\
\hline Sergipe & Fundação de Amparo à Pesquisa e Inovação do Estado de Sergipe \\
\hline Tocantins & Fundação de Amparo à Pesquisa do Estado do Tocantins \\
\hline
\end{tabular}

Fonte: http://confap.org.br/news/presidentes-das-faps, 2018.

\section{AGÊNCIAS E SISTEMAS DE AVALIAÇÃO DA PESQUISA}

A concepção de avaliação da pesquisa e da pós-graduação, na atualidade, requer visão integradora e multidimensional, pela qual se complementam diferentes métodos, ferramentas e modelos. Embora os estudos métricos da informação revistam-se de importância extraordinária para a tomada de decisões estratégicas institucionais, participando das políticas de gestão das atividades científicas e tecnológicas do país, os dados e informações com os quais trabalham devem ser interpretados em contextos discursivos, culturais, econômicos e políticos da produção de conhecimentos.

Com o incentivo à criação de cursos de pós-graduação no Brasil, na década de 1970, as políticas públicas foram influenciadas a fim de que as publicações científicas fossem fomentadas. Logo, na década de 1980, surgiram os primeiros programas de incentivo aos periódicos brasileiros, pelo CNPq e pela FINEP (CAPES, 2017).

A Avaliação do Sistema Nacional de Pós-Graduação (SNPG), essencial para assegurar e manter a qualidade dos cursos de mestrado e doutorado no país, surgiu em 1998, nos moldes em que se conhece hoje. Tal avaliação é orientada pela Diretoria de Avaliação da CAPES e 
realizada com a participação da comunidade acadêmico-científica, representada por consultores ad hoc. Ela objetiva, inicialmente por meio de uma metodologia trienal de avaliação, certificar a qualidade da pós-graduação brasileira (referência para a distribuição de bolsas e recursos para o incentivo à pesquisa). Hoje, esse sistema de avaliação é quadrienal e busca localizar as assimetrias regionais e de áreas estratégicas do conhecimento no SNPG, a fim de orientar ações para a criação e a expansão de programas de pós-graduação no território nacional (CAPES, 2017). Nesse processo, é essencial o envio de relatórios pelos programas de pós-graduação, contendo as informações sobre em quais periódicos os pesquisadores a eles vinculados mais publicaram dentro do triênio ou quadriênio analisado. O relatório e a coleta de dados são realizados de modo padronizado dentro da Plataforma Sucupira, também desenvolvida e sustentada pela CAPES.

A maioria dos periódicos nacionais é vinculada a programas de pós-graduação, já que os periódicos surgiram proximamente aos cursos como modo de dar vazão à produção científica desenvolvida nas instituições de ensino superior.

Essa relação entre periódicos e programas de pós-graduação promoveu o aumento dos títulos de revistas, tornando-se necessária a definição de parâmetros e indicadores que apontassem a relevância e a qualidade de uma publicação para a sua respectiva comunidade científica. Desse modo, esses instrumentos serviriam como um crivo de qualidade, tanto válido para os pesquisadores que ali publicassem quanto para os próprios periódicos e programas, que poderiam ser beneficiados com incentivos para continuarem se mantendo ativos.

A demanda por um controle de qualidade dos periódicos originou o mecanismo denominado Qualis, da CAPES, que, de modo geral, constitui o processo de avaliação de pósgraduação no país, visto que, conforme já dito, a maioria das revistas brasileiras está vinculada a cursos de pós-graduação.

As revistas constantes desses relatórios são classificadas pelas comissões científicas, categorizadas por áreas da CAPES, conforme estratificação que define a qualidade da produção intelectual dos periódicos e, consequentemente, dos programas e dos pesquisadores segundo algumas estratificações, conforme a Figura 2 (CAPES, 2017c).

Figura 2: Estratos de Avaliação do Qualis - CAPES.

\begin{tabular}{|c|c|c|c|c|c|}
\hline Qualis & \multicolumn{5}{|c|}{ Estratos } \\
\hline Internacional & A1 & A2 & & \\
\hline Nacional & B1 & B2 & B3 & B4 & B5 \\
\hline Nacional & C & & & & \\
\hline
\end{tabular}


Fonte: Elaborado pelos autores, 2018.

Legenda: azul: excelência internacional; amarelo: excelência nacional; salmão: relevância nacional média; verde: baixa relevância.

Com conceito A são contempladas as revistas de abrangência internacional; o conceito $\mathrm{B}$ é atribuído às revistas que têm como foco o público nacional; enquanto que o conceito $\mathrm{C}$, não recomendado, é atribuído às revistas de abrangência local. Cada comitê de área também apresenta critérios específicos para sua avaliação.

Dentre os critérios gerais tem-se, por exemplo: periodicidade, qualidade do corpo editorial da revista, sistema de avaliação por pares, normalização, indexação, diversidade de origens dos trabalhos, difusão e popularidade da revista e, em alguns casos, isto é, para alguma áreas como as Ciências Exatas e Biológicas considera-se o Fator de Impacto, baseado nos indicadores de citação gerados pelas bases indexadoras, como a do "Journal Citation Report" (JCR) do Institute for Scientific Information(ISI), mencionado na Unidade 3 sobre bases de dados e indicadores de impacto e visibilidade da ciência.

Há discussão polêmica sobre esses parâmetros de avaliação definidos pelos Documentos de Área da CAPES e que vêm sendo aplicados pelos pesquisadores, pois, em muitos casos, não correspondem à realidade das revistas brasileiras, como por exemplo, a exigência existente de que o periódico tenha visibilidade internacional por meio da indexação numa base de dados, o que nem sempre um periódico nacional considerado como de boa qualidade consegue galgar, devido às relações de poder e à estrutura e conjuntura do seu campo científico de origem (CAPES, 2017c).

Outra questão a ser levantada é que há um limite de periódicos para os estratos mais altos. Portanto, se há um número maior de revistas em condições de serem estratificadas com Qualis A1 e se não há espaço destinado para isso, algumas delas devem manter-se no estrato abaixo. Isso ocorre devido ao discutível critério de que a quantidade de revistas classificáveis como A1 (estrato mais alto) deve ser inferior ao número de revistas classificadas como A2.

Outro ponto que restringe ainda mais a possibilidade de as revistas serem classificadas no estrato A é que os periódicos A1 somados aos A2 devem atingir no máximo $25 \%$ do total de revistas da sua área do conhecimento, já o total de revistas $\mathrm{A} 1+\mathrm{A} 2+\mathrm{B} 1$ deve totalizar, no máximo, o valor de 50\% do total de periódicos de sua área. Logo, a soma B2+B3+B4+B5 representaria os outros $50 \%$, já que o ideal é que não existam revistas avaliadas com estrato C (KISHI, 2017). Observa-se, também, que não é obrigatório preencher todos os estratos, entretanto, as porcentagens não devem ultrapassar o exposto pela regra descrita. 
Pesquisas no domínio de estudos métricos da informação têm sido realizadas com o propósito de repensar esse sistema de avaliação desigual e aperfeiçoá-lo, de modo a propor metodologias e indicadores alternativos e complementares, a fim de refinar a atribuição de critérios e mais bem avaliar a pesquisa que se produz, relevando, desse modo, aspectos peculiares de cada área e ensejando que revistas de boa qualidade se internacionalizem por meio de sua presença em bases indexadoras e seus artigos possam ser reconhecidos e, portanto, mais citados e visíveis.

O foco na dimensão qualitativa da pesquisa, no sentido de contextualizar critérios quantitativos de modo mais aprofundado e de ponderar aspectos relacionados ao histórico de criação das áreas de pesquisa, bem como das tipologias de suas relações de colaboração científica, podem influenciar no estabelecimento de novos e mais equitativos instrumentos de avaliação.

\section{CONSIDERAÇÕES FINAIS}

Do exposto, foi possível reconhecer o cenário contemporâneo das Políticas de Ciência e Tecnologia no Brasil, desde o seu surgimento em meio a ações do Estado, que as fomentou como recurso estratégico de desenvolvimento do país.

Observa-se que o sistema de comunicação científica se mantém em decorrência de políticas e iniciativas de fomento por agências nacionais reguladoras que foram enunciadas ao longo da unidade. O papel do MCTI, do CNPq e da CAPES, especialmente no que tange à criação do sistema de avaliação atual da pesquisa científica, englobando a consolidação de periódicos e programas de pós-graduação, é fundamental para o estabelecimento de parâmetros de qualidade da pesquisa e sua consequente visibilidade internacional.

Contudo, estudos têm sido realizados no âmbito da Ciência da Informação para que esses parâmetros sejam aperfeiçoados e se tornem mais apropriados à avaliação das distintas áreas do conhecimento, levando-se em conta as possibilidades de internacionalização da pesquisa, já que a internacionalização é o critério que desponta como primordial para se ter uma boa avaliação conforme os mecanismos enunciados, atendendo desse modo, ao principal objetivo do Estado em relação à produção científica, de promover a internacionalização da pesquisa e o consequente desenvolvimento tecnológico e da inovação no país.

Em síntese, o processo de comunicação científica, desde as atividades de produção, organização, comunicação e divulgação da ciência, é regulado e consolidado pelas políticas científicas e tecnológicas vigentes. 


\section{REFERENCIAS}

ARAÚJO, V. M. R. H. A organização espacial da informação científica e tecnológica no Brasil. Ciência da Informação, Brasília, v. 14, n. 1, p. 17-24, jan./jun. 1985.

BRASIL. Ministério da Ciência, Tecnologia e Inovação (MCTI). Indicadores Nacionais de CT\&I: apresentação. Brasília, 2012a. Disponível em: <http://www.mct. gov.br/index.php/content/view/2043.html>. Acesso em: 20 jul. 2017.

Indicadores Nacionais de CT\&I: histórico. Brasília, 2012b. Disponível em: <http://www.mct.gov. br/index.php/content/view/2044/Historico.html>. Acesso em: 20 jul. 2017.

CAVAlCANTE, L. R. Políticas de Ciência, Tecnologia e Inovação no Brasil: uma análise com base nos indicadores agregados. Rio de Janeiro: Secretaria deAssuntos Estratégicos da Presidência da República, 2009. Disponível em: <http:// www.ipea.gov.br/portal/images/stories/PDFs/TDs/td_1458.pdf>. Acesso em: 31 jul. 2017.

COLE, S.; COLE, J. R. Visibility and the structural bases of awareness of scientific research. American Sociological Review, [S. 1.], v. 33, n. 3, p. 397-413, 1968.

CONSELHO NACIONAL DE DESENVOLVIMENTO CIENTÍFICO E TECNOLÓGICO (CNPq). Programas. 2017. Disponível em: 〈http://cnpq.br/\#void〉. Acesso em: 13 jul. 2017.

COORDENAÇÃO DE APERFEIÇOAMENTO DE PESSOAL DE NÍVEL SUPERIOR (CAPES). Portal de Periódicos. 2017a. Disponível em: <http://www.periodicos. capes.gov.br>. Acesso em: 26 jul. 2017a.

COORDENAÇÃO DE APERFEIÇOAMENTO DE PESSOAL DE NÍVEL SUPERIOR (CAPES). Prêmio Capes Teses. 2017b. Disponível em: 〈http://www.capes.gov.br/ premiocapesdetese>. Acesso em: 26 jul. $2017 \mathrm{~b}$.

Sobre a avaliação. 2017c. Disponível em: 〈http://capes.gov.br/avaliacao/sobre-a-avaliacao〉. Acesso em: 27 jul. 2017c.

DIAS, R. B. O que é a política científica e tecnológica. Sociologias, Porto Alegre, v. 13, n. 28, p. 316-344, set./dez. 2011.

DIAS, R. B. Sessenta anos de política científica e tecnológica no Brasil. Campinas: UNICAMP, 2012.

FREITAS, J. L.; BUFREM, L. S. As políticas públicas de informação expressas na produção científica brasileira em Ciência da Informação. In: ENCUENTRO DE LA ASOCIACIÓN DE EDUCACIÓN E INVESTIGACIÓN EN CIENCIA (EDICIC) DE LA INFORMACIÓN DE IBEROAMÉRICA Y EL CARIBE Patrimônio, mediaçõessociais e tecnologias, 10., 2016, Belo Horizonte. Anais... Belo Horizonte: UFMG, 2016.

FROHMANN, B. Taking information policy beyond information science: applying the actor network theory. In: ANNUAL CONFERENCE CANADIAN ASSOCIATION FOR INFORMATION SCIENCE, 23., 1995, Edmond. Proceedings... Alberta, 1995. Disponível em: <http://www.caisacsi.ca/proceedings/1995/ frohmann_1995.pdf>. Acesso em: 13 jul. 2017. 
GONZÁLEZ DE GÓMEZ, M. N. Novos cenários políticos para a informação. Ciência da Informação, Brasília, v. 31, n. 1, p. 27-40, jan./abr. 2002.

GONZÁLEZ DE GÓMEZ, M. N.; CANONGIA, C. (Org.). Contribuição para políticas de ICT. Brasília: MCT/IBICT, 2001.

HOWLETT, M.; RAMESH, M.; PERL, A. Políticas públicas: seus ciclos esubsistemas: uma abordagem integradora. Rio de Janeiro: Elsevier, 2013.

JARDIM, J. M.; SILVA, S. C. de A.; NHARRELUGA, R. S. Análise de políticas públicas: uma abordagem em direção às políticas públicas de informação. Perspectivas em Ciência da Informação, Belo Horizonte, v. 14, n. 1, p. 2-22, 2009.

KISHI, K. Qual é a do Qualis Periódicos? Conheça sua história e classificações. 2017. Disponível em: <https://galoa.com.br/blog/qual-e-do-qualis-periodicos-conheca-sua-historiae-classificacoes >. Acesso em: 25 jul. 2017.

LIMA, M. F. B. F. Produção científica: revisão parcial da literatura brasileira com ênfase na área agrícola. Ciência da Informação, Brasília, v. 22, n. 3, p. 233- 236, 1993.

PIRES-ALVES, F. Informação científica, educação médica e políticas de saúde: a Organização Pan-Americana da Saúde e a criação da Biblioteca Regional de Medicina BIREME. Saúde Coletiva, Rio de Janeiro, v. 13, n. 3, p. 900-908, 2008.

PROGRAMA CIÊNCIA SEM FRONTEIRAS. [Sobre]. 2017. Disponível em: <http://www. cienciasemfronteiras.gov.br/web/csf>. Acesso em: 26 jul. 2017.

REDE NACIONAL DE ENSINO E PESQUISA (RPN). A rede. 2017. Disponível em: $<$ https://memoria.rnp.br/en>. Acesso em: 28 jul. 2017.

SANTOS, M. M.; CARDOSO FILHO, J. C. Informação e políticas públicas: responsabilidade social da Ciência da Informação. Biblios, [S. 1.], n. 45, p. 29-39, 2011.

SILVA, E. M.; GARCIA, J. C. R. Política de Informação Científica e Tecnológica no Brasil. DataGramaZero: Revista de Ciência da Informação, Rio de Janeiro, v.15, n.4, ago. 2014.

SILVA, F. M. A informação científica e tecnológica brasileira no âmbito da sociedade da informação: uma análise das iniciativas governamentais. Revista Digital de Biblioteconomia e Ciência da Informação, Campinas, v. 2, n. 2, p. 18-30, jan./jun. 2005.

SOUZA, C. Políticas públicas: uma revisão de literatura. Sociologias, Porto Alegre, v. 8, n. 16, p. 20-45, jul./dez. 2006.

TAKAHASHI, T. (Org.). Sociedade da informação no Brasil: livro verde. Brasília: MCT, 2000. 